\title{
Screening of miRNA prognostic biomarkers of gastric adenocarcinoma and biological function analysis of their target genes
}

\section{Guoliang Zheng}

Cancer Hospital of China Medical University (Liaoning Cancer Hospital and Institute)

\section{Dong Yang}

Department of Pathophysiology, College of Basic Medical Science, China Medical University

\section{Yan Zhao}

Cancer Hospital of China Medical University (Liaoning Cancer Hospital and Institute)

Zhichao Zheng ( $\nabla$ drzhengzhichao1@163.com )

Cancer Hospital of China Medical University (Liaoning Cancer Hospital and Institute)

\section{Research Article}

Keywords: Stomach adenocarcinoma, TCGA database, MiRNA, Prognosis, Target genes

Posted Date: February 21st, 2022

DOI: https://doi.org/10.21203/rs.3.rs-1298081/v1

License: (c) (i) This work is licensed under a Creative Commons Attribution 4.0 International License. Read Full License 


\section{Abstract}

Objective To identify microRNA (miRNA) prognostic biomarkers of stomach adenocarcinoma (STAD).

Methods Bioinformatics analysis were performed by screening of miRNA sequencing and clinical data in TCGA-STAD database and identification of differentially expressed miRNAs (DEMs) using the R software package. The data were randomly divided (1:1) into a training set and a test set to construct a linear prognostic risk model of miRNAs for Cox regression analysis. To evaluate the predictive power of the prognostic model, Kaplan-Meier survival analysis and generation of receiver operating characteristic (ROC) curves were performed in the test group and all patients with gastric cancer, respectively. The miRNA-target genes were predicted using the prognostic model, and then their biological functions were analyzed.

Results A total of 267 DEMs were identified (185 upregulated, 82 downregulated). The reliability of DEMs in the model was verified by qPCR. Five miRNAs (miR-135b-3p, miR-196b-3p, miR-942-3p, miR-143-5p, and miR-9-3p) were identified as independent prognostic factors by univariate and multivariate COX analyses. According to Kaplan-Meier survival analysis, the overall survival rate in the high-risk group was lower than that in the low-risk group. A total of 2,498 miRNA target genes were predicted, mainly enriched in Notch, MAPK, and TGF-beta signaling pathways. qPCR analysis confirmed that the five DEMs in the model were consistent with bioinformatics analysis results of the DEMs in the TCGA-STAD database.

Conclusion The prognostic risk model with the five differentially expressed miRNAs can be used to predict the prognosis of STAD, which will help to further guide clinical treatment.

\section{Introduction}

Stomach adenocarcinoma (STAD) is one of the most common and deadly cancers. In 2018 , there were more than one million new cases of gastric cancer, and the disease caused an estimated 783,000 deaths worldwide[1]. Although advances in the diagnosis and treatment of STAD have been achieved in recent years, the overall prognosis of patients with STAD has improved only slightly[2]. Recently, the etiology of gastric cancer has been increasingly understood, such as information regarding its risk factors, including Helicobacter pylori infection, family history of upper gastrointestinal cancer, history of major gastric resection, smoking, salt intake, and food fumigation[3]. However, the pathogenesis of STAD has not been fully elucidated. Therefore, it is necessary to study the pathogenesis and progression of STAD in order to improve STAD treatment and patient survival. Although miRNAs were previously considered to lack the ability to encode proteins without a biological function, they have attracted more and more attention by researchers in recent years, due to advances in high-throughput sequencing techniques. It has been reported that miRNAs play important roles in a variety of tumors[4-7], including tumorigenesis, tumor development, and prognosis. Therefore, systematic research on specific miRNAs can provide a new reference for elucidating the pathogenesis of human malignant tumors, leading to the potential identification of novel biomarkers for disease diagnosis, treatment, and prognosis. 
In this study, we constructed and validated a prognostic risk model by analyzing miRNA sequencing data from The Cancer Genome Atlas (TCGA)-STAD database. The next steps were to predict the target genes of the identified miRNAs in our model and to analyze their biological functions. Finally, the reliability and effectiveness of the differentially expressed miRNAs in the model were verified by quantitative polymerase chain reaction (qPCR) analysis. The results of this study will further reveal the relationship between miRNAs and STAD as well as identify specific miRNAs that could be used as novel histopathological prognostic factors for decisions related to the individualized treatment of STAD patients.

\section{Methods And Materials}

\section{1 miRNAs and clinicopathological information}

The miRNAs and clinicopathological information were obtained from the TCGA-STAD database using the website: https://cancergenome.nih.gov/, with the following filters: (1) Primary Site, Stomach; (2) Project, TCGA-STAD; (3) Disease Type, adenomas and adenocarcinomas; (4) Data Category, transcriptome profiling; and (5) Data Type, Isoform Expression Quantification. The version of the miRNA data was 16.0, which was released on March 26, 2019, including information of 45 paracancerous tissues and 446 gastric cancer tissues. The downloaded data included the clinicopathological data of 443 cases. The ActivePerl (version 5.26, 64-bit) scripting language was used for the integration and extraction of the miRNA expression data and clinical data. R software (version 3.6.1) and the corresponding R package were used for data processing and analysis. This study complied with the relevant regulations on TCGA data access and patient privacy protection, although ethics committee approval was not required for data obtained from TCGA. All methods were performed in accordance with the relevant guidelines and regulations.

\subsection{Screening of the differentially expressed miRNAs}

The differentially expressed miRNAs in the TCGA-STAD database were identified by using the edgeR package in $\mathrm{R}$ software. The selection criteria were log2 (fold change) greater than 1.5 and a false discovery rate less than 0.05 . The volcano plot of the differentially expressed miRNAs was drawn by the ggplot2 package of $\mathrm{R}$ software.

\subsection{Establishment and evaluation of the prognostic risk model}

In this study, the STAD patients were randomly assigned to a training set or a test set at a 1:1 ratio. The risk ratio (HR) calculated from the univariate Cox regression model of the training set was used to identify overall survival (OS)-related miRNAs, and miRNAs with $\mathrm{P}<0.05$ were selected as candidate miRNAs for biomarkers. The miRNA candidates were incorporated into the multivariate Cox regression model for further analysis. The importance score of each miRNA was calculated by supervised principal component analysis (PCA); then the important miRNAs were selected by the criterion of 10-fold cross-validation. 
These miRNAs were used to eventually construct a prognostic risk model based on their expression levels. The following formula was used for the prognostic score calculation: Prognostic score $=(\beta 1 \times$ expression level of gene 1$)+(\beta 2 \times$ expression level of gene2 $)+(\beta n \times$ expression level of gene $n)$.

The miRNA prognostic model was established based on analysis of the training set data, and this model was further used to calculate the prognostic scores of the test set and all patients with gastric cancer. The median prognosis score of the training set was used as a critical value to separate the high-risk group and the low-risk group of the training set, test set, and all STAD patients. The existence of survival differences between the high- and low-risk groups was verified by Kaplan-Meier survival curve analysis. The predictive power of the prognostic risk model was evaluated by the time-dependent receiver operating characteristic (ROC) curve. The prognostic risk model and clinical parameters were incorporated into either univariate or multivariate Cox survival analysis for evaluation of the prognostic value of the independent prognostic model. Univariate and multivariate Cox survival analyses were visualized by forest maps. All of the analytical data mentioned above were generated using the related packages in $\mathrm{R}$ software, such as survminer, caret, glmnet, survival, and survival ROC.

\subsection{Prediction of miRNA-target genes using the prognostic risk model and construction of the miRNA-mRNA co- expression network}

The prediction of miRNA target genes was completed by analysis using three different databases (miRDB, miRTarBase, and TargetScan). To reach a high prediction accuracy, the target gene candidates were selected if they were found in two or more databases. The Venn diagram was drawn using the VennDiagram package of $\mathrm{R}$ software.

\subsection{Biological function analysis of target genes}

Analysis of the biological function of miRNA target genes was performed using Gene Ontology (GO), which is a major bioinformatics tool for annotating genes and gene products. $\mathrm{GO}$ contains terms under three categories: (1) cellular component, (2) molecular function, and (3) biological process. Another tool for biological function analysis is KEGG (Kyoto Encyclopedia of Genes and Genomes), which is a database resource for understanding high-level functions and utilities of biological systems. The results of $\mathrm{GO}$ and KEGG analyses were presented using several packages of R software, including BiocManager, clusterProfiler, enrichplot, ggplot2, colorspace, and stringi. For comparisons, $\mathrm{P}<0.05$ was considered statistically significant for determining the true difference among groups.

\subsection{Cell culture}

GSE-1, a SV40-transformed human fetal gastric epithelial cell line, and BGC-823, a gastric cancer cell line, were routinely cultured with high-glucose Dulbecco's modified Eagle medium containing $10 \%$ fetal bovine serum, penicillin $(100 \mathrm{U} / \mathrm{mL})$, and streptomycin $(100 \mu \mathrm{g} / \mathrm{mL})$ and maintained in a $5 \% \mathrm{CO}_{2}$ cell 
incubator. The culture medium was changed every two to three days. When the cell density reached $90 \%$ confluency, cell passaging was conducted at a 1:2 ratio.

\section{7 qPCR}

Total cellular RNA was isolated using the Trizol reagent (Invitrogen, USA). RNA was reverse-transcribed into CDNA using the miRNA First Strand cDNA Synthesis Kit (Sangon Biotech, China). Verification of the differentially expressed miRNAs identified by informatics analysis was performed using an miRNA qPCR Kit (Sangon Biotech, China) and the ABI7500 system (Applied Biosystems, USA). The small nuclear RNA U6 served as an internal reference for the miRNA PCR. Each sample was analyzed three times for each detection indicator, and the collected threshold cycle $(\mathrm{Ct})$ value was averaged. The relative expression of the identified miRNAs was calculated by the $2^{-\triangle \triangle C t}$ method. The primer sequences used in this study were as follows: miR-135b-3p-Forward, UUUGUUCGUUCGGCUCGCGUGA; miR-135b-3p-Reverse, ACGCGAGCCGAACGAACAAAUU; miR-143-5p-Forward, GCGCAGCGCCCTGTCTCCCAG; miR-143-5pReverse, GCTGCAGAACAACTTCTCTC; miR-196-3p-Forward, GCGTAGGTAGTTTCCTG; miR-196-3pReverse, GAGCAGGCTGGAGAA; miR-942-3p-Forward, GCCAGATCTTGATTGACTTACAGCCCAGTT; miR942-3p-Reverse, GCCGAATTCCACCTGTCTTTATTCCACCC; miR-9-3p-Forward, GAGGCCCGTTTCTCTCTTTG; miR-9-3p-Reverse, AGCTTTATGACGGCTCTGTG; and U6-Forward, CTCGCTTCGGCAGCACA; U6-Reverse, AACGCTTCACGAATTTGCGT.

\section{Results}

\subsection{Flow diagram}

The flow diagram of our project is presented in Fig. 1.

\section{2 miRNA data and clinicopathological information obtained from the TCGA-STAD database}

In the TCGA-STAD database, excluding the 54 patients whose total survival time was less than 1 month, a total of 389 STAD patients were enrolled into this study, including 255 males (mean age: $64.98 \pm 10.71$ years) and 134 females (mean age: $66.02 \pm 10.77$ years). The recruited patients were randomly divided into two groups: a training set $(n=194)$ and a test set $(n=195)$. No statistically significant difference in clinical covariates between the two groups was found, as shown in Table $1(P>0.05)$. 
Table 1

Clinical characteristics of the patients enrolled in this study.

\begin{tabular}{|c|c|c|c|c|c|}
\hline Clinical characteristics & $\begin{array}{l}\text { Total } \\
(n=389)\end{array}$ & $\begin{array}{l}\text { Training set } \\
(n=194)\end{array}$ & $\begin{array}{l}\text { Test set } \\
(n=195)\end{array}$ & $\chi^{2}$ value & $\begin{array}{l}P \\
\text { value }\end{array}$ \\
\hline \multirow[t]{2}{*}{ Age at diagnosis (years) } & $65.1(30-88)$ & 64.9 & 65.3 & 1.074 & 0.622 \\
\hline & & $(30-86)$ & $(34-88)$ & & \\
\hline Gender & & & & 0.032 & 0.859 \\
\hline Male & 255 & 127 & 128 & & \\
\hline Female & 134 & 68 & 66 & & \\
\hline Histologic Grade & & & & 1.498 & 0.478 \\
\hline Well & 8 & 3 & 5 & & \\
\hline Moderate & 138 & 74 & 64 & & \\
\hline Poor & 234 & 113 & 121 & & \\
\hline Stage & & & & 7.826 & 0.069 \\
\hline I & 49 & 28 & 21 & & \\
\hline II & 122 & 60 & 62 & & \\
\hline III & 164 & 84 & 80 & & \\
\hline IV & 45 & 14 & 31 & & \\
\hline $\mathrm{T}$ & & & & 5.36 & 0.252 \\
\hline T1 & 18 & 11 & 7 & & \\
\hline $\mathrm{T} 2$ & 85 & 41 & 44 & & \\
\hline T3 & 179 & 88 & 91 & & \\
\hline T4 & 101 & 53 & 48 & & \\
\hline $\mathrm{N}$ & & & & 0.511 & 0.475 \\
\hline No & 115 & 61 & 54 & & \\
\hline $\mathrm{N}+$ & 263 & 129 & 134 & & \\
\hline Distant metastasis & & & & 2.88 & 0.091 \\
\hline M0 & 349 & 181 & 168 & & \\
\hline M1 & 26 & 9 & 17 & & \\
\hline
\end{tabular}


As shown in Fig. 2, based on the comparison of miRNA data between the gastric cancer tissue samples $(n=446)$ and the paracancerous tissues $(n=45)$, a total of 267 differentially expressed miRNAs were identified, including 185 upregulated miRNAs (69.3\%) and 82 downregulated miRNAs (30.7\%) in gastric cancer.

\subsection{Establishment and evaluation of the prognostic risk model}

To construct a prognostic risk model, both univariate and multivariate Cox regression analyses of the training set were performed in this study. This model was composed of five differentially expressed miRNAs in STAD. The following formula was created based on the analysis of the model: Prognostic risk score $=(-0.1484 \times$ miR-135b-3p expression level $)+(0.1345 \times$ miR-143-5p expression level $)+(-0.3068 \times$ miR-196b-3p expression level) + (-0.1783 × miR-942-3p expression level) + (0.1474 × miR-9-3p expression level). As shown in Table 2, two miRNAs (miR-143-5p and miR-9-3p, HR value > 1) were associated with a high risk of STAD (high expression, increased risk of death of the patient), and three miRNAs (miR-135b$3 p$, miR-196b-3p, and miR-942-3p, HR value < 1) were protective against STAD (high expression, decreased risk of death of the patient).

Table 2

Prognostic risk model constructed by $\mathrm{R}$ software.

\begin{tabular}{|llllll|}
\hline miRNA & Coef & HR & HR.95L & HR.95H & P value \\
\hline hsa-miR-135b-3p & -0.148 & 0.862 & 0.706 & 1.052 & 0.144 \\
\hline hsa-miR-143-5p & 0.134 & 1.144 & 0.961 & 1.361 & 0.129 \\
\hline hsa-miR-196b-3p & -0.306 & 0.735 & 0.526 & 1.027 & 0.072 \\
\hline hsa-miR-942-3p & -0.178 & 0.837 & 0.663 & 1.055 & 0.132 \\
\hline hsa-miR-9-3p & 0.147 & 1.159 & 0.988 & 1.358 & 0.069 \\
\hline Note: Coef, regression coefficient; HR, risk ratio. & & \\
\hline
\end{tabular}

As shown in Fig. 3, analysis found that in the training set, patients belonging to the high-risk group had a higher mortality rate than those in the low-risk group. Similar results were also found in the test set and in all patients.

As shown in Fig. 4, Kaplan-Meier analysis of the training set $(n=194)$ showed that the 3-year survival rate of the patients in the high-risk group was significantly lower than that in the low-risk group, with a statistically significant difference $(P=1.417 \mathrm{e}-4)$. Similar results were also found in the test set and all STAD patients. These results suggest that the prognostic risk model composed of the five differentially expressed miRNAs can be used to predict the 3-year survival of STAD patients. The ROC curves for the three groups, including the training set, the test set, and all STAD patients, were relatively smooth, and the area under the curve (AUC) was greater than 0.7 , indicating that the model had a good prognostic performance. 
As shown in Fig. 5, univariate Cox regression analysis showed that the following factors, including the primary tumor size, tumor stage, node stage, metastasis, tumor/lymph node/metastasis stage, and risk score model with the five miRNAs, were significantly correlated with the prognosis $(P<0.05)$. Further multivariate Cox regression analysis revealed that the risk score model with the five miRNAs was an independent prognostic factor for STAD $(H R=1.971, P<0.001)$.

\subsection{Venn diagram and miRNA-mRNA regulatory network}

As shown in Fig. 6, the target genes of the five differentially expressed miRNAs were predicted by three different databases (miRDB, miRTarBase, and TargetScan) (Fig. 6A-E). Analysis of the miRNA-mRNA network showed that the five differentially expressed miRNAs and their target genes are closely related to each other (Fig. 6F).

\subsection{GO enrichment and KEGG analyses}

As shown in Fig. 7, GO enrichment and KEGG analyses revealed that miRNA target genes were enriched mainly in the G1/S phase transition of the cell cycle, Notch signaling pathway, RNA polymerase II proximal promoter sequence-specific DNA binding, and proximal promoter sequence-specific DNA binding.

\subsection{Verification of the five differentially expressed miRNAs found in the TCGA-STAD database}

As shown in Table 3 and Fig. 8, the five differentially expressed miRNAs found in the TCGA-STAD database that were included in the model were verified by qPCR using RNA samples collected from either GSE-1 cells or BGC-823 cells. The results showed that compared to normal GSE-1 cells, miR-143-5p and miR-9-3p were downregulated; while miR-135b-3p, miR-196b-3p, and miR-942-3p were upregulated in gastric cancer BGC-823 cells.

Table 3

Verification of the five differentially expressed miRNAs in gastric cancer by qPCR.

\begin{tabular}{|llll|}
\hline Name & GSE-1 cells & BGC-823 cells & P value \\
\hline hsa-miR-135b-3p & $1.353 \pm 0.118$ & $4.898 \pm 0.325$ & $<0.05$ \\
\hline hsa-miR-143-5p & $3.595 \pm 0.134$ & $1.782 \pm 0.089$ & $<0.05$ \\
\hline hsa-miR-196b-3p & $1.241 \pm 0.086$ & $5.529 \pm 0.378$ & $<0.05$ \\
\hline hsa-miR-942-3p & $2.356 \pm 0.098$ & $3.987 \pm 0.156$ & $<0.05$ \\
\hline hsa-miR-9-3p & $4.256 \pm 0.169$ & $1.997 \pm 0.079$ & $<0.05$ \\
\hline
\end{tabular}

\section{Discussion}


In this study, the differentially expressed miRNAs in STAD were identified by bioinformatics technology using the TCGA-STAD database. The differentially expressed miRNAs were then integrated with clinical parameters to establish a prognostic risk model, which was composed of the five differentially expressed miRNAs. This model was shown to have a good prognostic performance in the training set, test set, and all patients, as the AUC of the ROC curve was greater than 0.7 , indicating a moderate accuracy when it was used to predict the 3-year survival. More importantly, multivariate Cox regression analysis further demonstrated that this model could independently predict the prognosis of STAD. Therefore, the five differentially expressed miRNAs included in this model can be used as a biomarker for the prediction of OS of STAD patients. Based on the analysis of the miRNA target genes and their enrichment pathways, several gastric cancer-related signaling pathways were found, including the Notch[8], mitogen-activated protein kinase[9], and transforming growth factor-beta[10] signaling pathways. Furthermore, our study found a potential role of our prognostic risk model in the molecular pathogenesis, clinical progression, and prognosis of gastric cancer, which is likely to aid in the preventive diagnosis and individualized treatment of gastric cancer.

Among the five differentially expressed miRNAs mentioned above, two of them (miR-143-5p and miR-9$3 p$ ) indicated a high risk of STAD (their expression level was negatively correlated with the OS). They have been considered as tumor-suppressor genes in recent years, such as miR-143-5p in lung cancer[11], esophageal cancer[12], pancreatic cancer[13], gallbladder cancer[14], and osteosarcoma[15]; and miR-9$3 p$ in bladder cancer[16], liver cancer[17], nasopharyngeal cancer[18], and breast cancer[19]. However, there is no related report about the functions of miR-143-5p and miR-9-3p in human gastric cancer. The results of the data from the TCGA-STAD database indicated that both of them have a low expression level in STAD. Our experiments also found that the expression level of both of these miRNAs in the gastric cancer cell line was lower than that in the normal gastric cell line. In our prognostic model, the cases with highly expressed miR-143-5p and miR-9-3p had a positive correlation with a poor prognosis. Therefore, the specific mechanism of the role of these two miRNAs in gastric cancer still needs to be further clarified.

Meanwhile, the three other miRNAs, including miR-135b-3p, miR-196b-3p, and miR-942-3p, indicated a low risk of STAD (the expression level was positively correlated with the OS). Currently, the specific mechanism of these three miRNAs has been rarely studied, and their correlation with gastric cancer also needs further study. It is believed that with further in-depth studies, the biological role and potential mechanism of these miRNAs will become clearer.

According to ROC curve analysis, the AUC value of our prognostic risk model was greater than 0.7 (between 0.7 and 0.9 ) in both the training group and the test group, indicating that the prognostic model had a moderate accuracy for the diagnosis of STAD. Univariate and multivariate Cox regression analyses revealed that this model could independently predict the OS $(H R=1.971, P<0.001)$.

However, our study still has some shortcomings, such as the fact that the random assignment of data came from a single database into the training set and the test set. A separate set of external tests should 
be created to make the results more convincing. Second, the follow-up time of the TCGA-STAD study cohort was relatively short (the average follow-up time was only 20.78 months), and the exclusion rate was relatively high, which may reduce the reliability of the Kaplan-Meier analysis method. In the future, it is necessary to recruit more STAD patients and to conduct longer follow-up studies in order to verify the findings of this study. In addition, the complex effects and specific mechanisms of these differentially expressed miRNAs in STAD need to be further investigated.

\section{Conclusions}

In summary, our results suggest that the prognostic risk model with five differentially expressed miRNAs could significantly differentiate the groups of patients with different prognoses of STAD in either the training set or the test set, when predicting the 3-year OS. Therefore, this model may be used as a novel biomarker for evaluation of the prognosis of STAD patients. It is necessary to further study its value in clinical applications.

\section{Abbreviations}

AUC = Area Under the Curve; Coef = Regression coefficient; FDR = False Discovery Rate; $G 0=$ Gene Ontology; HR = Hazard Ratio; KEGG = Kyoto Encyclopedia of Genes And Genomes; LogFC = Log2 Fold Change; miRNA = microRNA; OS = Overall Survival; STAD = Stomach Adenocarcinoma; qPCR = Quantitative Polymerase Chain Reaction; ROC = Receiver Operating Characteristic; TCGA = The Cancer Genome Atlas.

\section{Declarations}

Ethics approval and consent to participate: Not applicable

Consent for publication: Not applicable

Availability of data and materials: All data generated or analysed during this study are included in this published article. The data that support the findings of this study can be found in the TCGA database: https://cancergenome.nih.gov/

Competing interests: The authors declare that they have no competing interests.

Funding: This study was supported in part by grants from Cultivation fund project of National Natural Science Foundation of China (No. 2021-ZLLH-06) and Medical engineering cross research fund project of Liaoning Province (No. LD202014)

Authors' contributions: ZGL made substantial contributions to the conception and the design of the study. YD and ZGL contributed to data acquisition, data analysis, and interpretation of the results. ZY and ZZC contributed to the drafting of the article and critically revising it for important intellectual content. All 
authors agree to be accountable for all aspects of the work and ensuring that questions related to the accuracy or integrity of the work are appropriately investigated and resolved. All authors have read and approved the final manuscript.

Acknowledgements: We would like to thank the Pathophysiology Department of China Medical University for providing the cell lines and assisting with the cell culture.

\section{Authors' information (optional)}

ORCID

Zhi-Chao Zheng https://orcid.org/0000-0001-6841-1672

\section{References}

1. Bray F, Ferlay J, Soerjomataram I, Siegel RL, Torre LA, Jemal A: Global cancer statistics 2018: GLOBOCAN estimates of incidence and mortality worldwide for 36 cancers in 185 countries. CA: a cancer journal for clinicians 2018, 68(6):394-424.

2. Lazăr DC, Tăban S, Cornianu M, Faur A, Goldiş $A$ : New advances in targeted gastric cancer treatment. World journal of gastroenterology 2016, 22(30):6776-6799.

3. den Hoed CM, Kuipers EJ: Gastric Cancer. How Can We Reduce the Incidence of this Disease? Current gastroenterology reports 2016, 18(7):34.

4. Kong W, He L, Richards EJ, Challa S, Xu CX, Permuth-Wey J, Lancaster JM, Coppola D, Sellers TA, Djeu JY et al: Upregulation of miRNA-155 promotes tumour angiogenesis by targeting VHL and is associated with poor prognosis and triple-negative breast cancer. Oncogene 2014, 33(6):679-689.

5. Li BS, Zuo QF, Zhao YL, Xiao B, Zhuang Y, Mao XH, Wu C, Yang SM, Zeng H, Zou QM et al: MicroRNA25 promotes gastric cancer migration, invasion and proliferation by directly targeting transducer of ERBB2, 1 and correlates with poor survival. Oncogene 2015, 34(20):2556-2565.

6. Mokutani Y, Uemura M, Munakata K, Okuzaki D, Haraguchi N, Takahashi H, Nishimura J, Hata T, Murata K, Takemasa I et al: Down-Regulation of microRNA-132 is Associated with Poor Prognosis of Colorectal Cancer. Annals of surgical oncology 2016, 23(Suppl 5):599-608.

7. Wang K, Liang Q, Wei L, Zhang W, Zhu P: MicroRNA-608 acts as a prognostic marker and inhibits the cell proliferation in hepatocellular carcinoma by macrophage migration inhibitory factor. Tumour biology: the journal of the International Society for Oncodevelopmental Biology and Medicine 2016, 37(3):3823-3830.

8. Yao $\mathrm{Y}, \mathrm{Ni}$ Y, Zhang J, Wang $\mathrm{H}$, Shao S: The role of Notch signaling in gastric carcinoma: molecular pathogenesis and novel therapeutic targets. Oncotarget 2017, 8(32):53839-53853.

9. Ma W, Xu Z, Wang Y, Li W, Wei Z, Chen T, Mou T, Cheng M, Luo J, Luo T et al: A Positive Feedback Loop of SLP2 Activates MAPK Signaling Pathway to Promote Gastric Cancer Progression. Theranostics 2018, 8(20):5744-5757. 
10. Li N, Xie C, Lu NH: Transforming growth factor- $\beta$ : an important mediator in Helicobacter pyloriassociated pathogenesis. Frontiers in cellular and infection microbiology 2015, 5:77.

11. Sanada H, Seki N: Involvement of Dual Strands of miR-143 (miR-143-5p and miR-143-3p) and Their Target Oncogenes in the Molecular Pathogenesis of Lung Adenocarcinoma. 2019, 20(18).

12. Yang $C$, Shen $S$, Zheng $X$, Ye K, Sun $Y, L u Y, G e H$ : Long noncoding RNA HAGLR acts as a microRNA143-5p sponge to regulate epithelial-mesenchymal transition and metastatic potential in esophageal cancer by regulating LAMP3. FASEB journal: official publication of the Federation of American Societies for Experimental Biology 2019, 33(9):10490-10504.

13. Liu C, Wang JO, Zhou WY, Chang XY, Zhang MM, Zhang Y, Yang XH: Long non-coding RNA LINC01207 silencing suppresses AGR2 expression to facilitate autophagy and apoptosis of pancreatic cancer cells by sponging miR-143-5p. Molecular and cellular endocrinology 2019, 493:110424.

14. He M, Zhan M, Chen W, Xu S, Long M, Shen H, Shi Y, Liu Q, Mohan M, Wang J: MiR-143-5p Deficiency Triggers EMT and Metastasis by Targeting HIF-1a in Gallbladder Cancer. Cellular physiology and biochemistry: international journal of experimental cellular physiology, biochemistry, and pharmacology 2017, 42(5):2078-2092.

15. Yu X, Hu L, Li S, Shen J, Wang D, Xu R, Yang H: Long non-coding RNA Taurine upregulated gene 1 promotes osteosarcoma cell metastasis by mediating HIF-1a via miR-143-5p. Cell death \& disease 2019, 10(4):280.

16. Cai H, Yang X, Gao Y, Xu Z, Yu B, Xu T, Li X, Xu W, Wang X, Hua L: Exosomal MicroRNA-9-3p Secreted from BMSCs Downregulates ESM1 to Suppress the Development of Bladder Cancer. Molecular therapy Nucleic acids 2019, 18:787-800.

17. Tang J, Li Y, Liu K, Zhu Q, Yang WH, Xiong LK, Guo DL: Exosomal miR-9-3p suppresses HBGF-5 expression and is a functional biomarker in hepatocellular carcinoma. Minerva medica 2018, 109(1):15-23.

18. Ding Y, Pan Y, Liu S, Jiang F, Jiao J: Elevation of MiR-9-3p suppresses the epithelial-mesenchymal transition of nasopharyngeal carcinoma cells via down-regulating FN1, ITGB1 and ITGAV. Cancer biology \& therapy 2017, 18(6):414-424.

19. Zawistowski JS, Nakamura K, Parker JS, Granger DA, Golitz BT, Johnson GL: MicroRNA 9-3p targets $\beta 1$ integrin to sensitize claudin-low breast cancer cells to MEK inhibition. Molecular and cellular biology 2013, 33(11):2260-2274.

\section{Figures}




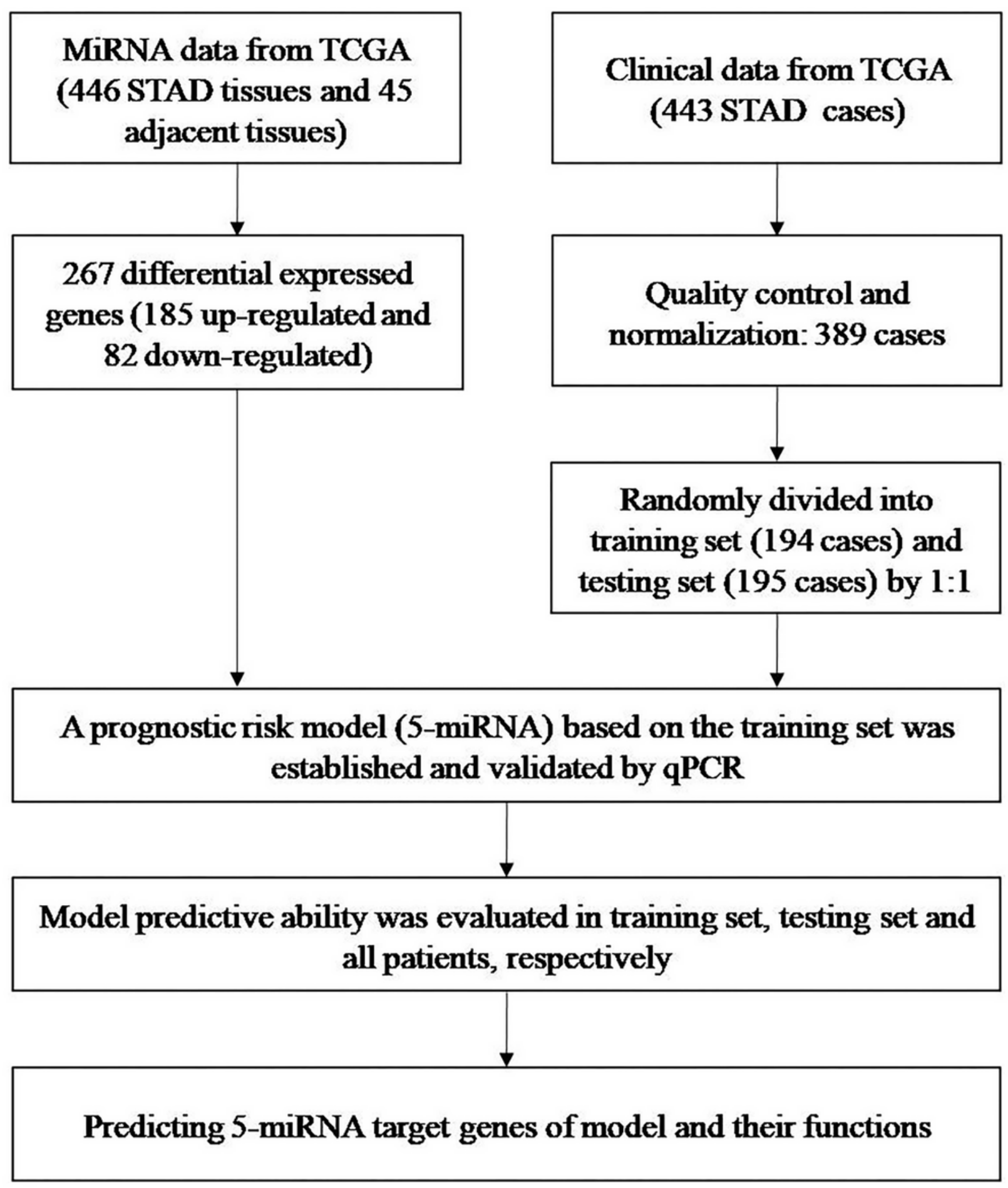

Figure 1

Flow diagram of this study. Differentially expressed genes (upregulated and downregulated) were randomly divided 1:1 into a training set (194 cases) and a test set (195 cases). The predictive ability of the model was evaluated in the training set. 


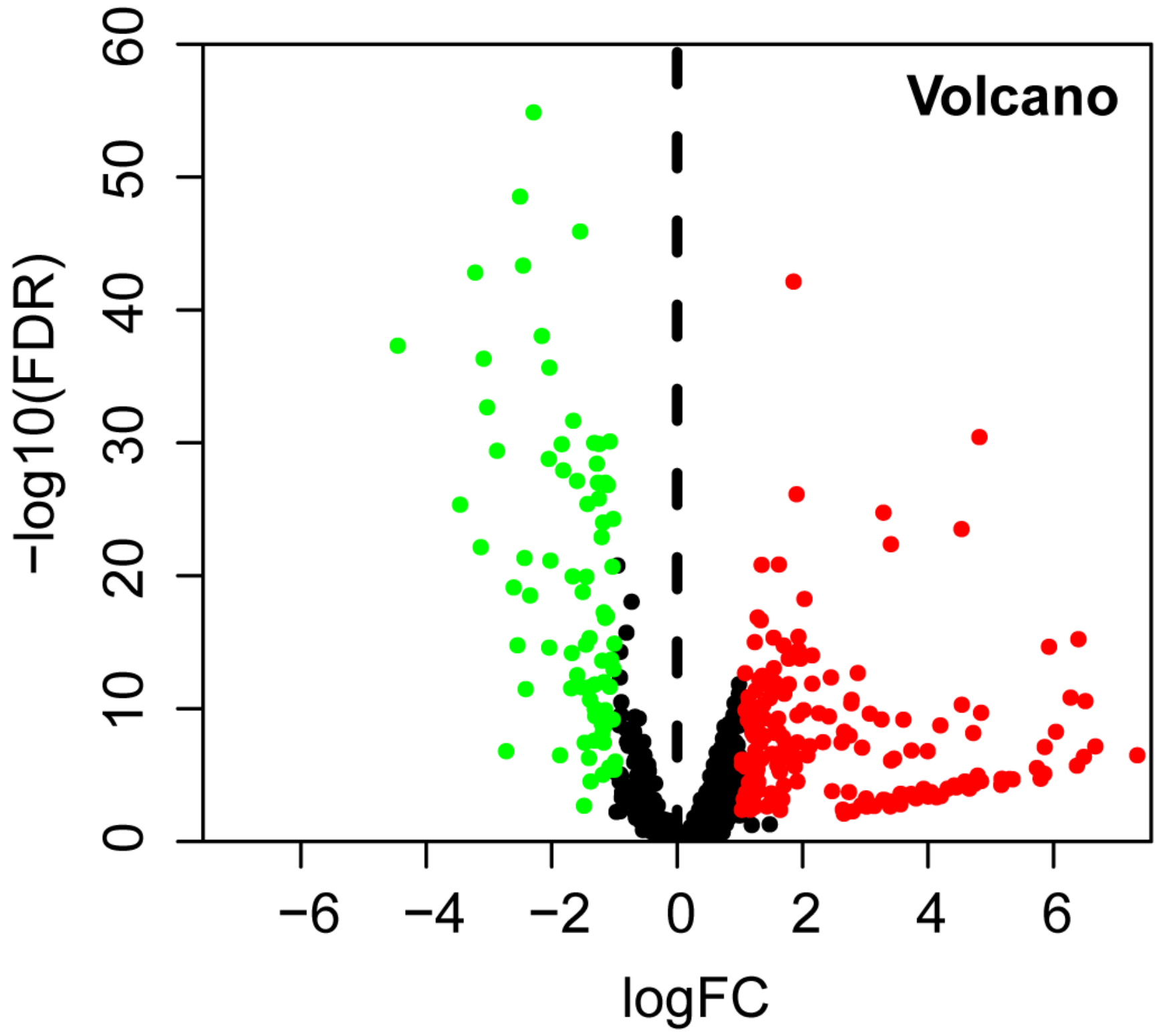

Figure 2

The volcano plot of the differentially expressed miRNAs in STAD. The upregulated miRNAs $(\log F C>1, P<$ $0.05)$ are marked in red, and the downregulated miRNAs ( $\log F C<-1, P<0.05)$ are marked in green. FDR, false discovery rate. 
A

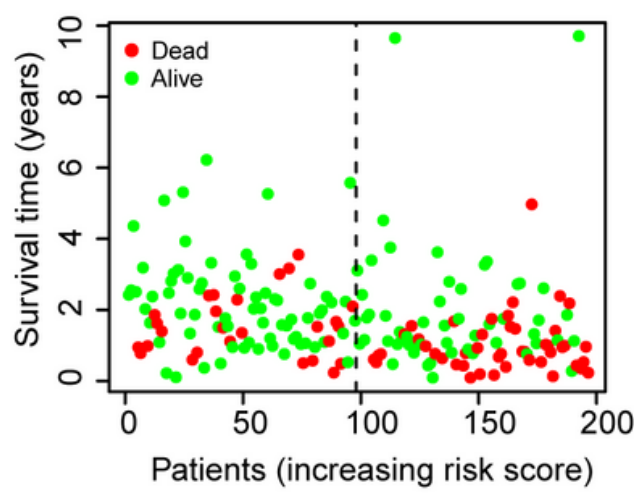

B

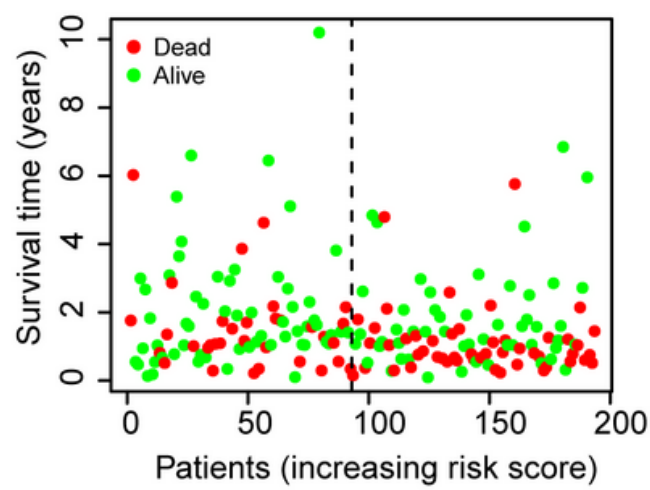

C

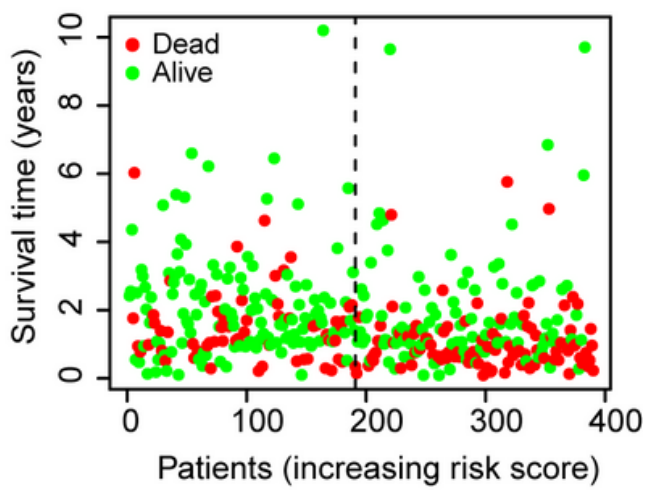

\section{Figure 3}

The time distribution of the survival status of the patients and the survival time. A. Training set; $\mathrm{B}$. Test set; C. All sets. Note: The abscissa indicates the cumulative frequency, and the ordinate represents the survival time (months). The green circles represent alive patients, and the red circles represent dead patients. The dotted lines represent the single inflection point of the risk score curve; therefore, the STAD patients were divided into a low-risk group and a high-risk group. 
A

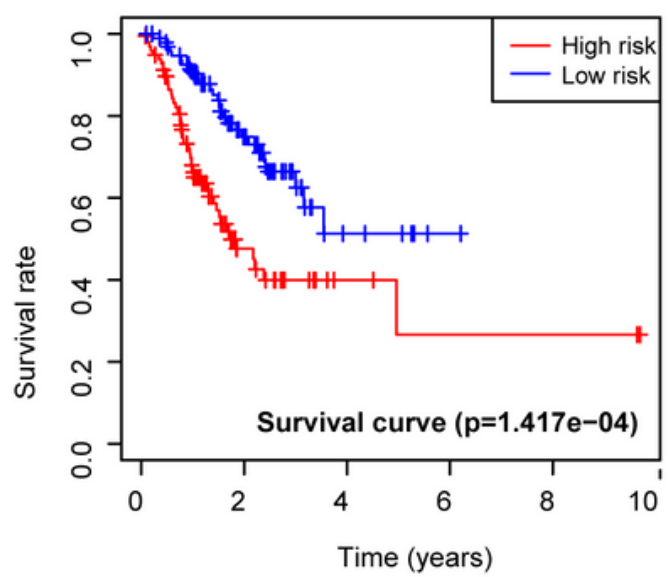

C

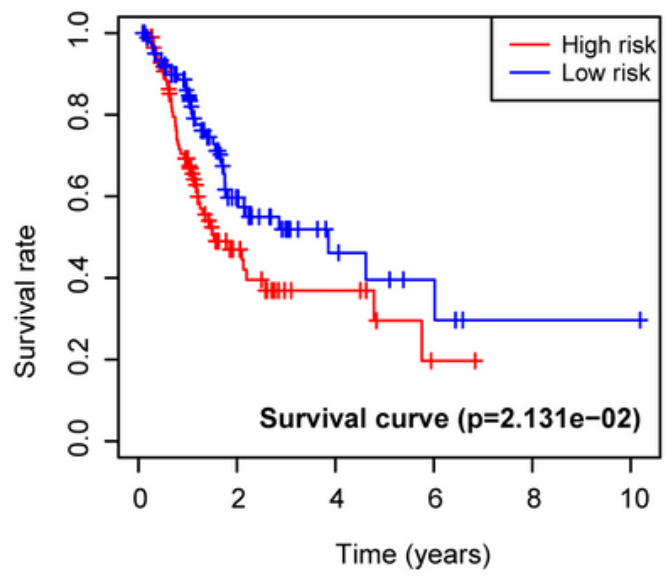

E

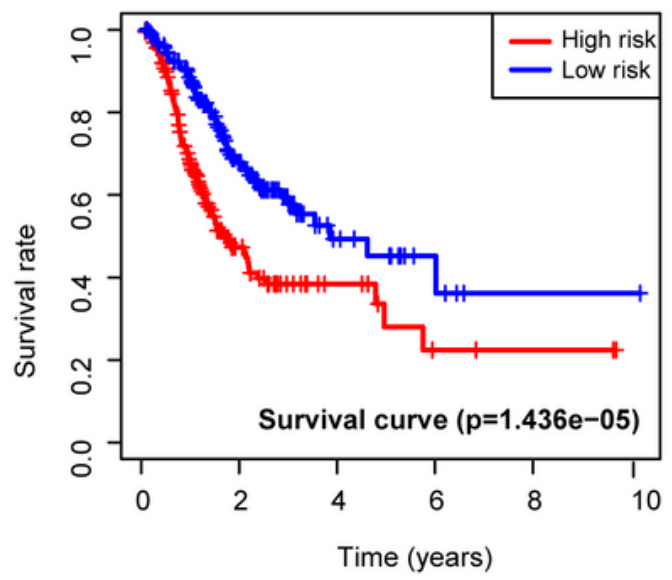

B

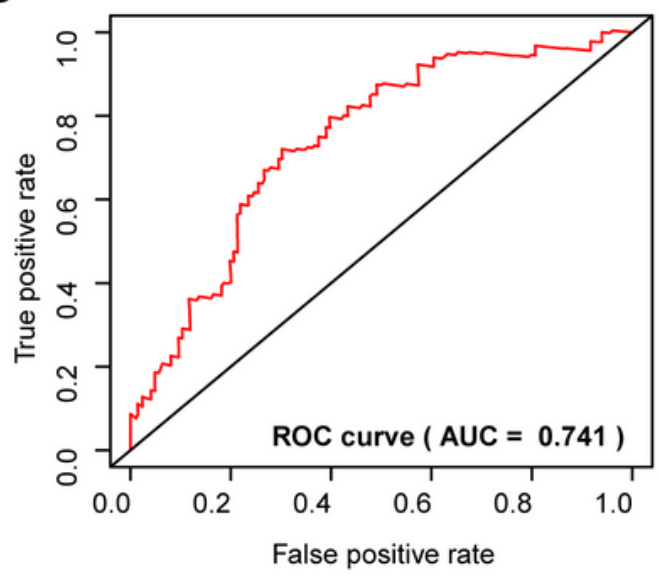

D

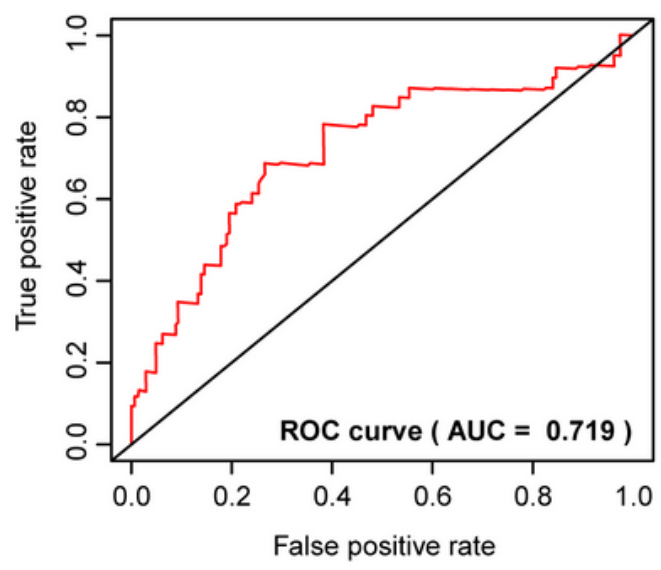

F

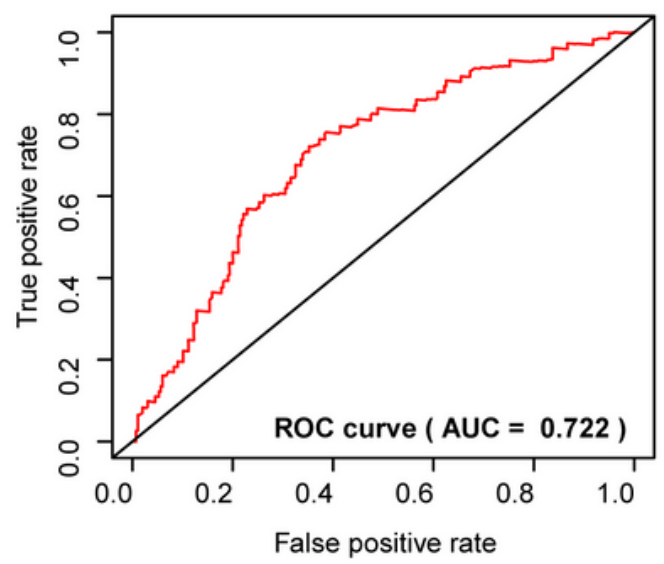

Figure 4

Kaplan-Meier survival curves and ROC curves of the three groups, based on analysis of the prognostic model. A, B. Training set; C, D. Test set; E, F. All sets. Note: In panels A, C, and E, the abscissa represents the overall survival (years), the ordinate represents the survival rate (\%), and the "+" represents deletion. In panels $B, D$, and $F$, the abscissa represents the true positive rate (sensitivity), and the ordinate represents the false positive rate (1-specificity). 
A

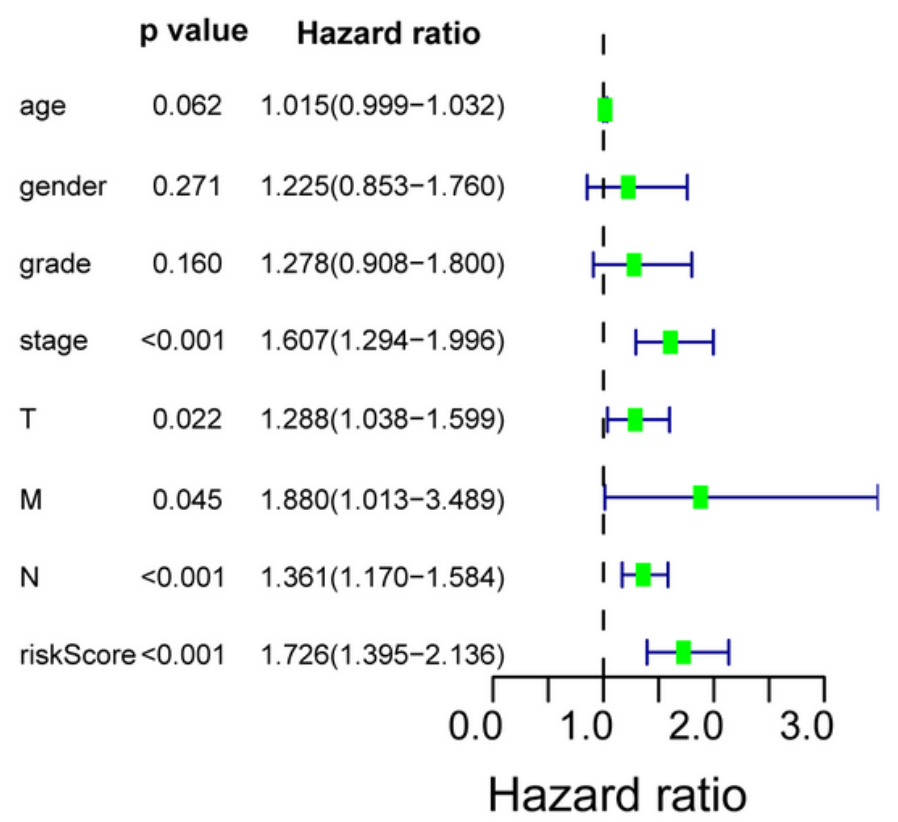

B

\begin{tabular}{lccc|} 
& p value & Hazard ratio \\
age & 0.002 & $1.027(1.010-1.045)$ \\
gender & 0.036 & $1.510(1.027-2.218)$ \\
grade & 0.550 & $1.115(0.781-1.591)$ \\
stage & 0.357 & $1.210(0.807-1.815)$ \\
$T$ & 0.186 & $1.215(0.911-1.621)$ \\
$\mathrm{M}$ & 0.127 & $1.818(0.844-3.917)$ \\
$\mathrm{N}$ & 0.065 & $1.233(0.987-1.540)$ \\
riskScore & $<0.001$ & $1.971(1.557-2.494)$ & 1 \\
& & 0 & 1 \\
& & Hazard ratio
\end{tabular}

\section{Figure 5}

Univariate and multivariate analyses of overall survival of patients. 


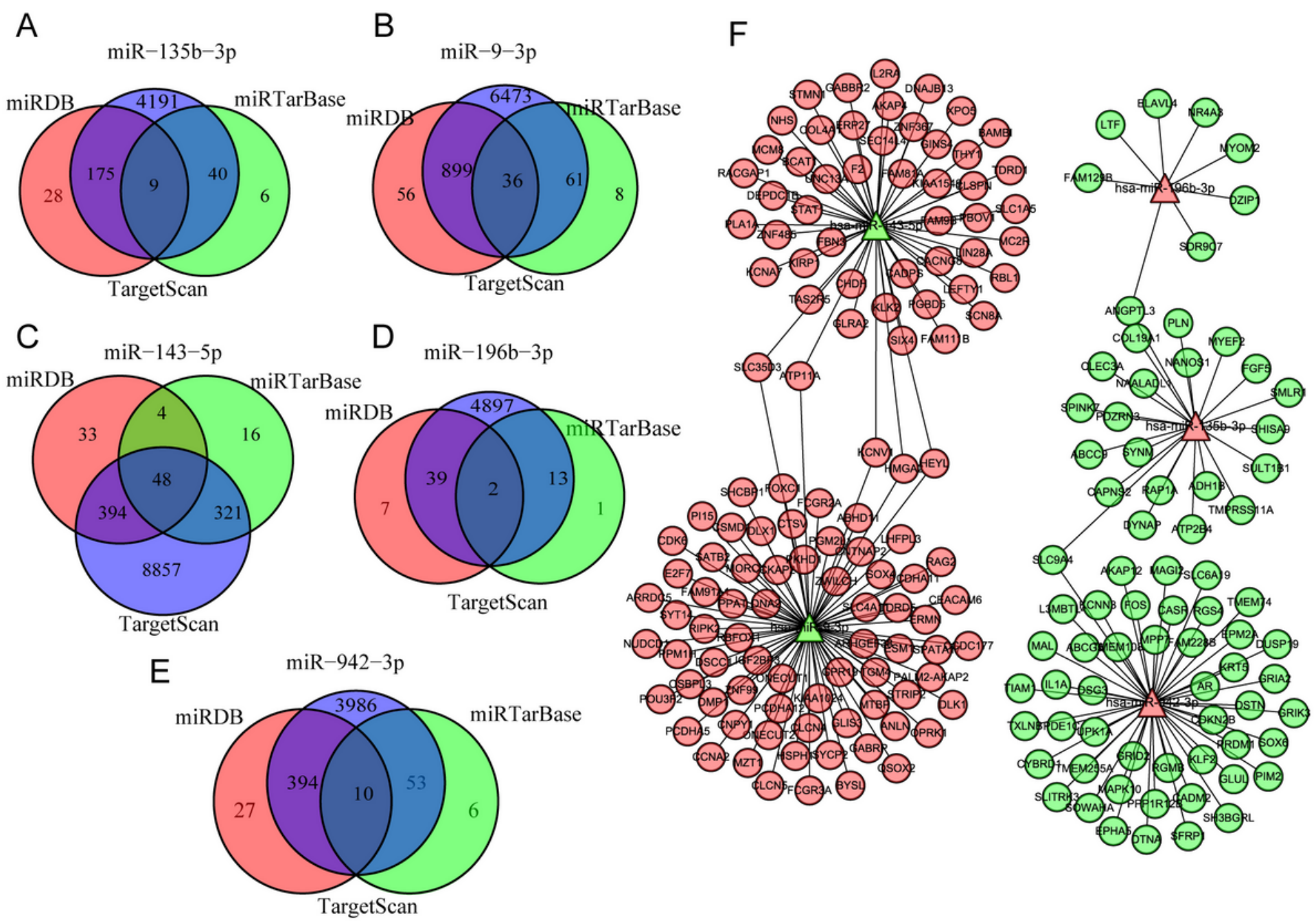

\section{Figure 6}

Venn diagram of the integrated analysis of three different databases and the results of the miRNA-mRNA co-expression network analysis. A. miR-143-5p; B. miR-9-3p; C. miR-135b-3p; D. miR-196b-3p; and E. miR942-3p. F. In the miRNA-mRNA regulatory network, red represents upregulation, blue represents downregulation, triangles represent miRNAs, and circles represent mRNAs. 
A

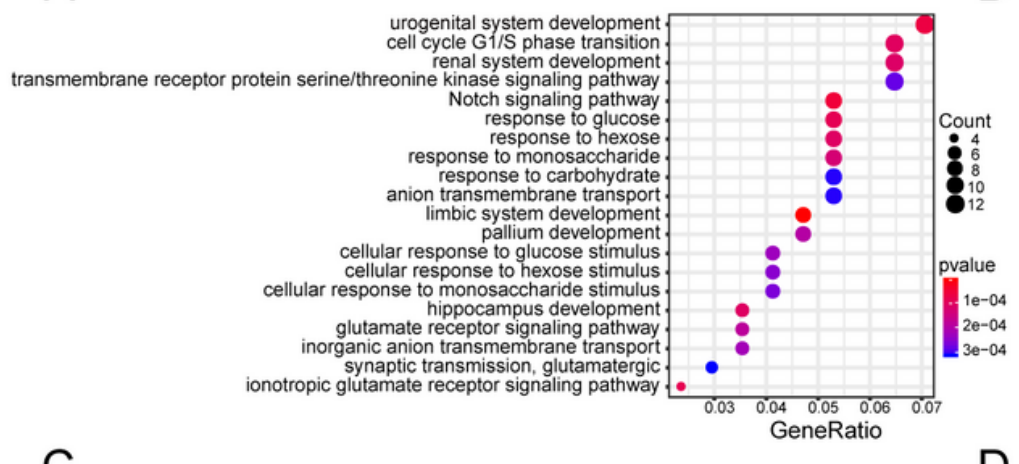

\section{C}

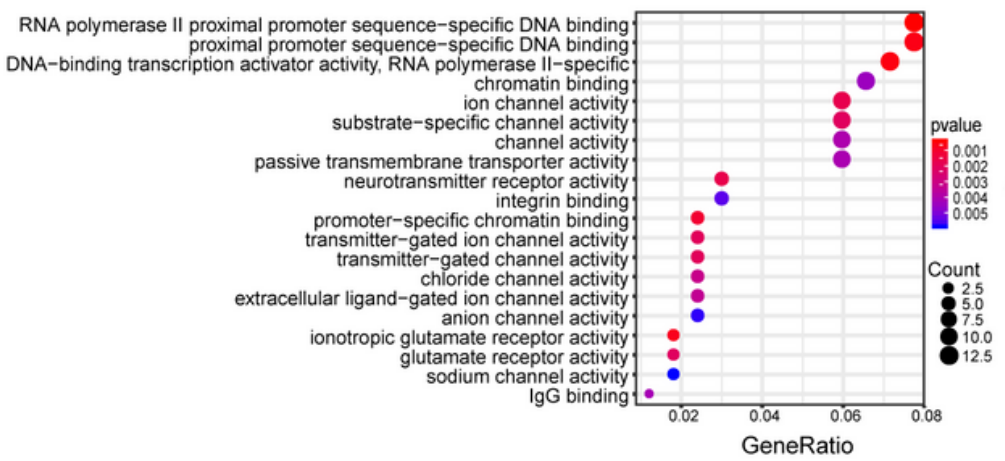

B
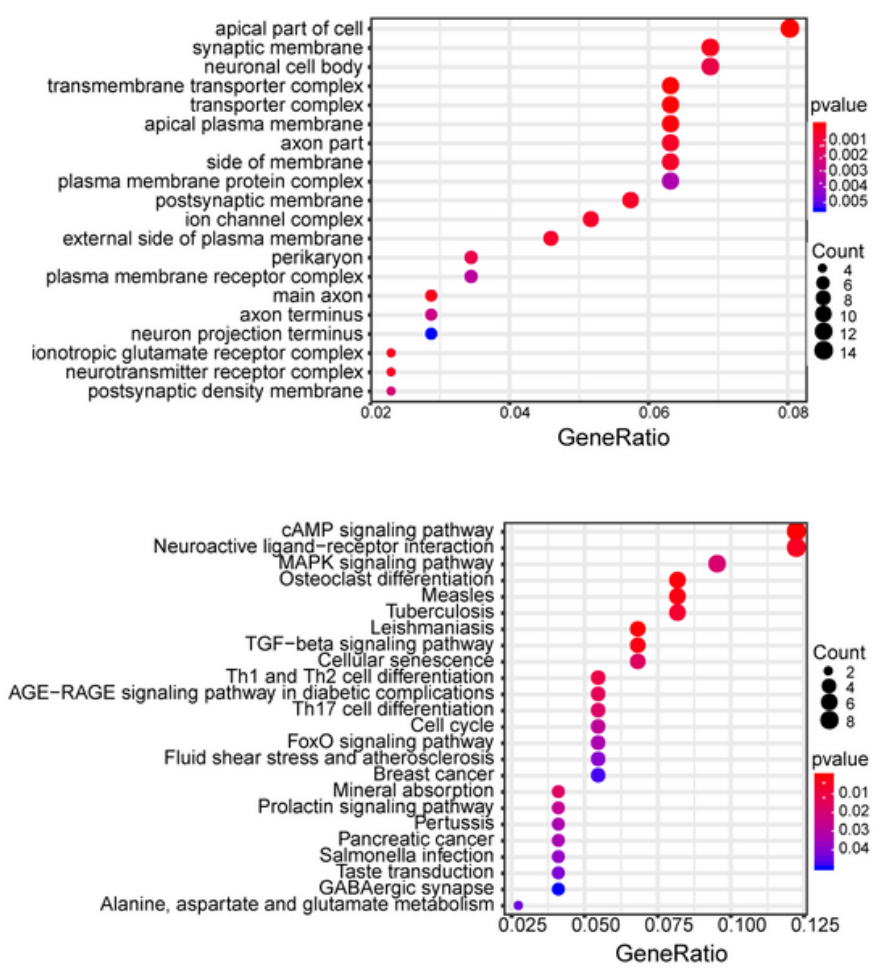

\section{Figure 7}

The results of GO enrichment and KEGG pathway analyses of miRNA target genes. A. Biological process; B. Cell composition; C. Molecular function; and D. KEGG pathways. GO, Gene Ontology; KEGG, Kyoto Encyclopedia of Genes and Genomes. The size of the circle represents the number of genes in the pathway, and the color intensity represents the -log10 (FDR) value. 


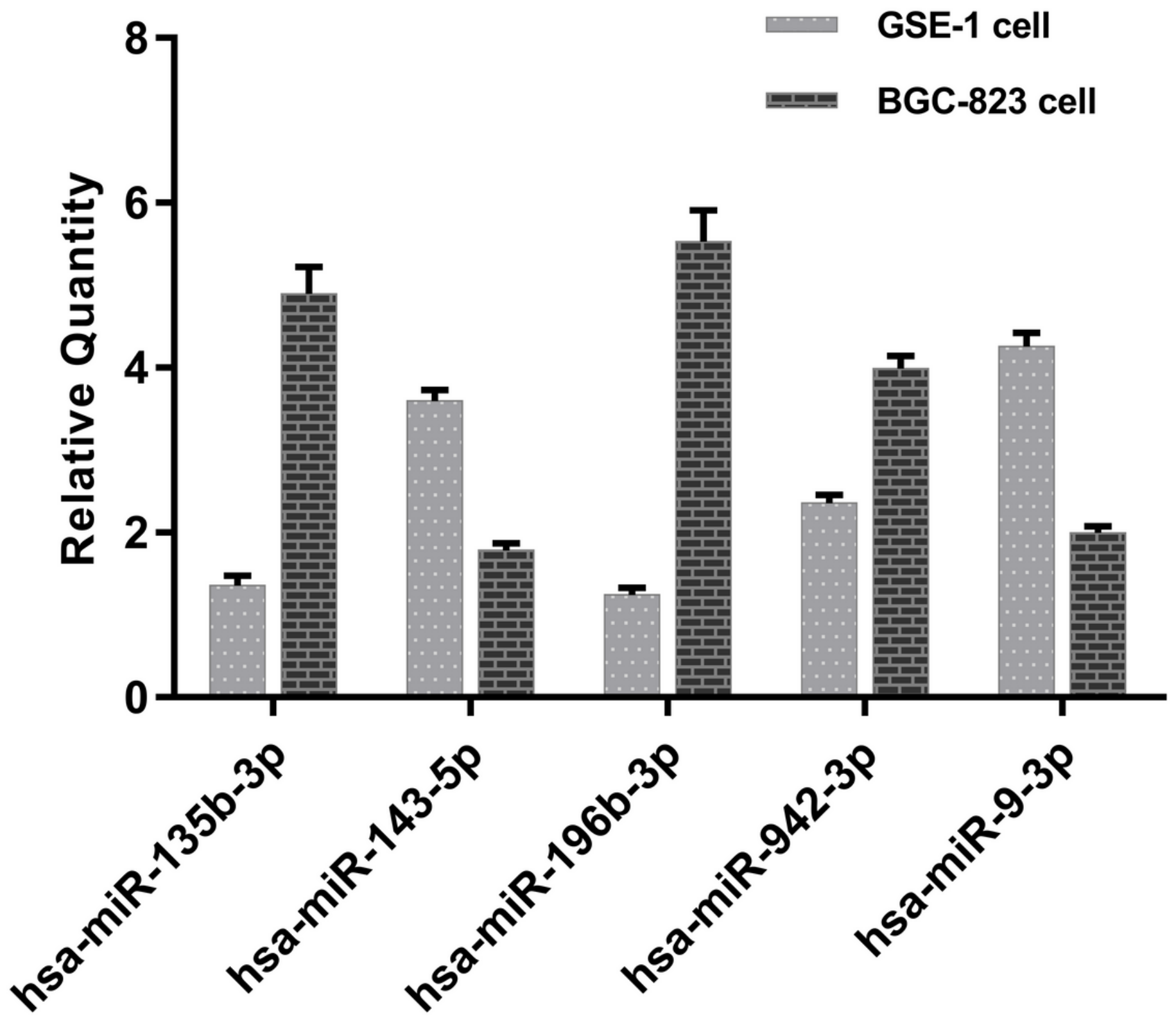

Figure 8

The relative expression levels of the five miRNAs in GSE-1 and BCG-823 cells. 